\title{
New Environmentally Friendly Dust Suppressant Based on Lignocellulosic Biomass from Wood Processing Wastewater
}

\author{
Sanita Vitolina ${ }^{1}$, Galia Shulga ${ }^{1}$, Brigita Neiberte ${ }^{1}$, Skaidrite Reihmane ${ }^{2}$, Elina Zilinska ${ }^{1}$ \\ ${ }^{1}$ Latvian State Institute of Wood Chemistry, 27 Dzerbenes St., Riga, Latvia \\ ${ }^{2}$ Riga Technical University, 3 Paula Valdena St., Riga, Latvia
}

\begin{abstract}
In this work, the possibility of usage of lignocellulosic biomass derived from wood processing wastewater as an environmentally friendly dust suppressant was studied. To increase the efficiency of the recovery of lignocellulosic biomass, a new developed composite coagulant, representing a polymer-colloid complex of polyethyleneimine with polyvalent metal ions, was applied. The effectiveness of the composite coagulant was examined using a model solution simulating the wastewater of hydrothermal treatment of birch wood. The optimum content of PEI in the composite coagulant was found to be $25-35 \%$. At the optimal composite coagulant dosage and $\mathrm{pH}$ value, the yield of the total wood biomass achieved 97\%, but the extraction of lignin and lignin-like substances was more than 65\%. Due to the polymeric and polyfunctional nature, the recovered wood biomass had glue properties. Taking into account the fact that the dust at the surface of unpaved roads poses considerable environmental problems, the biomass was tested as a structuring agent for sandy and model sandy-clay soils. The obtained results have shown that the separated lignocellulosic biomass was capable of forming large sandy aggregates that were able to decrease the dusty soil blowing off from the unpaved road surface.
\end{abstract}

Keywords: coagulation, composite coagulant, dust suppressant, lignocellulosic biomass, wastewater.

\section{INTRODUCTION}

In industrial veneer production, it is common to soak logs at elevated temperature prior to peeling. This hydrothermal pre-treatment is carried out to soften the logs and to ease the cutting during the peeling process [1]. The conditions of soaking depend on the wood species, the manufacturer, and the season of soaking. In Nordic and Eastern European countries, hydrothermal treatment is conducted at temperatures of $40-70^{\circ} \mathrm{C}$ for $8-48 \mathrm{~h} \mathrm{[2]}$. The obtained wastewater contains both suspended and dissolved substances, consisting of hemicelluloses, wood extractives, and lignin and its derivatives. Along with the strengthened public environmental protection awareness, the wastewater discharge regulations all over the world are becoming increasingly strict. Therefore, research on new technologies for wastewater treatment and the improvement of the existing ones becomes increasingly important.

Coagulation/flocculation is an important and established process in water treatment for removing contamination. In this process, coagulants are added to aggregate destabilized dissolved organic matter and colloidal particles into larger-sized flocs that can be effectively removed in subsequent separation processes [3]. Coagulants may be classified into inorganic and organic coagulants. High-charge cations, such as $\mathrm{Fe}^{3+}$ or $\mathrm{Al}^{3+}$, are among the most effective reagents for destabilizing the colloids. Therefore, common inorganic coagulants $\left(\mathrm{AlCl}_{3}\right.$, $\left.\mathrm{Al}_{2}\left(\mathrm{SO}_{4}\right)_{3}, \mathrm{FeCl}_{3}, \mathrm{Fe}_{2}\left(\mathrm{SO}_{4}\right)_{3}\right)$ and hydrolyzing metal salts based on aluminum or iron are widely used [4]. The polymers used in coagulation/flocculation treatment are commonly synthetic polyacrylamides, polyacrylic acids and polystyrene sulfonic acids, and their derivatives [5], [6]. To achieve better coagulation efficiency and floc characteristics, in recent years, research has been focused on the development of composite inorganic-organic coagulants. Composite materials pose tremendous potential in treating wastewater due to their better performance compared to that of conventional inorganic-based coagulants, and its lower cost than that of organic-based flocculants [7].

Sludge is a huge source of renewable organic matter that can be considered for sustainable resource recovery. Chemical compositions of the sludge depend on the wastewater quality, the nature of the chemicals used and the treatment processes involved. The results of several studies have indicated that chemical compositions are important while selecting potential reuse options [8]. Hence, prior knowledge of the physical and chemical properties of the sludge is necessary to reuse and recycle it into safe and sustainable disposal alternatives. Depending on the sludge chemical properties, it can be incorporated in 
brick and ceramic production [9], [10], used as a raw material for concrete and cement production [11], [12] and in manufacturing lightweight aggregates [13]. Sludge can be used as a low cost adsorbent for removal of contaminants (phosphorus, hydrogen sulfide, fluorides, perchlorate, and glyphosate) and heavy metals from wastewater [14], [15]. It is also applied in agricultural practice and other land based uses for soil structure improvement and $\mathrm{pH}$ adjustment [16], [17].

The aim of this work was to study the possibility of using the lignocellulosic biomass derived from wood processing wastewater as an environmentally friendly dust suppressant. To increase the efficiency of the recovery of lignocellulosic biomass, we used a new developed composite coagulant and investigated the chemical composition and yield of the precipitated biomass sludge depending on the environmental $\mathrm{pH}$ and the applied dosage of the coagulant.

\section{MATERIALS AND METHODS \\ Materials}

The wastewater of the hydrothermal treatment in veneer production was simulated with a model solution obtained by hydrothermal treatment of birch sawdust. The hydrothermal treatment of sawdust was performed with $0.01 \mathrm{M} \mathrm{NaOH}$ at a $1 / 50$ (mass ratio of the oven dry sawdust to water) hydromodulus and a temperature of $90^{\circ} \mathrm{C}$ for $4 \mathrm{~h}$. After hydrolysis, the hydrolyzate was separated from the sawdust treated through filtration. The model solution characterization has been published in our previous work [18].

In this study, a new composite coagulant (CC), representing a polymer-colloid complex of polyethyleneimine (PEI, Fluka, molecular mass of $750 \mathrm{kDa}$ ) with polyvalent metal ions, was developed. The polymer-colloid complex was formed due to the donor-acceptor interactions between imine atoms and metal cations. Besides, the complex was stabilized by $\mathrm{H}$-linkages, taking into account the hydration shell around the metal ions. The PEI/metal salt mass ratio in the $\mathrm{CC}$ varied from $0.16 / 1$ to $1 / 1$.

In order to evaluate the obtained results, we chose the early developed composite coagulant [19] for treatment of the model solution as the control.

\section{Experimental Procedure}

The coagulation process was carried out by way of mixing the model wastewater solution and the solution of the $\mathrm{CC}$ at a ratio of $1: 1$ and stirring the suspension with a magnetic stirring bar at $200 \mathrm{rpm}$ for $1 \mathrm{~min}$, followed by slow mixing at $40 \mathrm{rpm}$ for 2 min. The CC was added in the range of $20-140 \mathrm{mgl}^{-1}$ to the model solution. To characterize the coagulation performance of the $\mathrm{CC}$ at different $\mathrm{pH}$ values, experiments were conducted at $\mathrm{pH}$ values ranging from 5 to 8 by addition of $\mathrm{HCl}$ or $\mathrm{NaOH}$. Experiments were conducted in the temperature range of $13-60^{\circ} \mathrm{C}$ using a thermostat. The effectiveness of the coagulation was defined after a system settling time of $120 \mathrm{~min}$ and filtration. The residual concentration of the biomass and lignin was defined by measuring the obtained filtrate's optical density (A) at 490 and $280 \mathrm{~nm}$ using the previously obtained correlation curves for the biomass and lignin. Zeta potential and size measurements were conducted by Malvern Nanosizer SZ (UK).

To fractionate the lignocellulosic biomass and calculate the content of lignin and hemicelluloses therein, the precipitation of the hydrolyzate with concentrated sulfuric acid, with the following treatment of the formed filtrate with ethanol according to Liu and co-authors [20], was employed.

To provide useful information concerning the structure of the biomass sludge, analytical pyrolysis (Py-GC/MS) was applied. The analytical pyrolysis was performed with a Shimadzu GCMS-QP2010 coupled to a Frontier pyrolyzer.

The microstructure of the separated biomass surface was examined by scanning electron microscopy (SEM), using a scanning electron microscope (Tesla, Czech Republic).

Soil structuring experiments were conducted using powdered sand/clay model soil with a clay fraction content varied from 0 to $70 \%$. The clay fraction for this study was taken from the Lielauces quaternary clay deposit (Latvia). Its mechanical composition is shown in Table 1.

Table 1

\begin{tabular}{cccccccc}
\multicolumn{7}{c}{ Average grain size distribution (wt.\%) } \\
\hline Sampl & $>25$ & $250-$ & $50-$ & $20-$ & $10-5$ & $5-2$ & $<2$ \\
e & 0 & 50 & 20 & 10 & $\mu \mathrm{m}$ & $\mu \mathrm{m}$ & $\mu \mathrm{m}$ \\
& $\mu \mathrm{m}$ & $\mu \mathrm{m}$ & $\mu \mathrm{m}$ & $\mu \mathrm{m}$ & & & \\
\hline Clay & 2.3 & 9.6 & 14.3 & 12.1 & 13.4 & 15.5 & 34.5 \\
\hline
\end{tabular}

The sand was taken from the Baltic Sea cost and had the following mechanical composition: $3.1 \%$ of particles ranging from 1.0 to $0.25 \mathrm{~mm}, 93.9 \%$ of particles ranging from 0.25 to $0.05 \mathrm{~mm}$, and $3.0 \%$ of particles less than $0.01 \mathrm{~mm}$ in size.

In the work, the fraction of soil particles less than $0.25 \mathrm{~mm}$ was used. The soil aggregates $(>0.25 \mathrm{~mm})$ were obtained by mixing the soil with 1.0-5.0 $\mathrm{gdl}^{-1}$ biomass water suspensions. The used biomass had a gel form with the content of dry matter less than $10 \%$ and was obtained by centrifugation of the precipitated biomass sludge at $6000 \mathrm{rpm}$ during $20 \mathrm{~min}$ without its drying. The water suspensions with the defined biomass concentration were prepared by intensive mixing the biomass gel with water at $100 \mathrm{rpm}$ for 10 min using a mechanical mixer. The content of the biomass in the soil samples varied from $0.2 \%$ to $0.8 \%$ on their dry matter. The fractional composition of the dried structured soil was determined by dry sieving. The structuring coefficients $\mathrm{K} 1$ and $\mathrm{K} 2$ were found as the mass ratios of the sum of artificial aggregates > $0.25 \mathrm{~mm}$ to the initial sandy soil (K1) and of the sum 
of aggregates $>3.0 \mathrm{~mm}$ to the sum of all aggregates formed in the structured soil after the treatment (K2).

To determine the effect of wind on blowing off the treated soil particles depending on their size, a wind tunnel of original construction at a wind velocity of 5-25 ms ${ }^{-1}$ was applied. A certain mass of the soil sample was placed in the tunnel, and was subjected to the air flow with a variable speed for one minute. The loss of the mass was expressed as the percentage of the total soil mass.

\section{RESULTS AND DISCUSSION}

Isolation of Wood Biomass by Coagulation

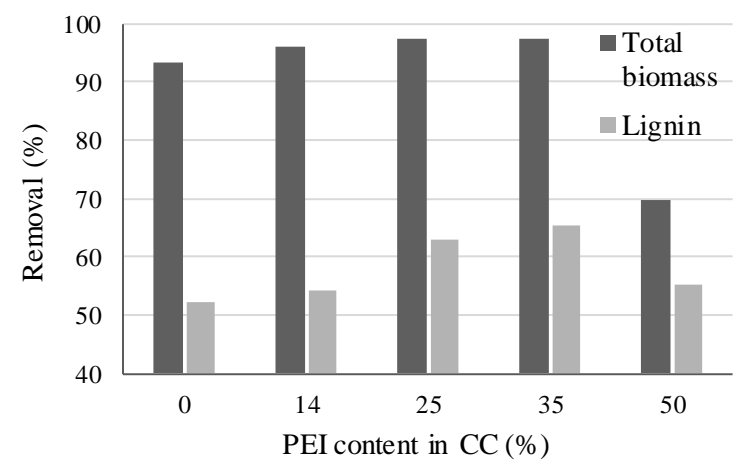

Fig. 1. Total biomass and lignin removal efficiency depending on the PEI content in $\mathrm{CC}\left(\mathrm{pH} 6\right.$, dosage $\left.100 \mathrm{mgl}^{-1}\right)$.

Fig. 1 shows the total biomass and lignin removal as a function of the PEI content in the $\mathrm{CC}$. The increase of the PEI content in the $\mathrm{CC}$ results in improving the efficiency of coagulation and particularly in lignin removal. The best results for the biomass separation occur by using the $\mathrm{CC}$ containing 25-35\% PEI in its composition. An increase in the PEI content by more than $35 \%$ leads to a rapid decrease in the biomass removal efficiency.

For the further study, we used the new developed composite coagulant consisting of $25 \%$ and $35 \%$ PEI.

The results of the total biomass and lignin removal efficiency depending on the $\mathrm{CC}$ and control coagulant dosage at pH 6.0 are shown in Fig. 2. The developed CC demonstrates an overwhelmingly high coagulation efficiency compared with the control coagulant at a given dosage range. The lignocellulosic biomass removal at a dosage of the CC more than $70 \mathrm{mgl}^{-1}$ is enhanced to a lesser extent. At the same time, the maximal lignin removal with CC-35 and CC-25 is achieved at the dosage close to $100 \mathrm{mg} \mathrm{l}^{-1}$. With the further increase in the dosage of the coagulant more than $100 \mathrm{mgl}^{-1}$, the removal efficiency of the biomass and lignin is decreased.
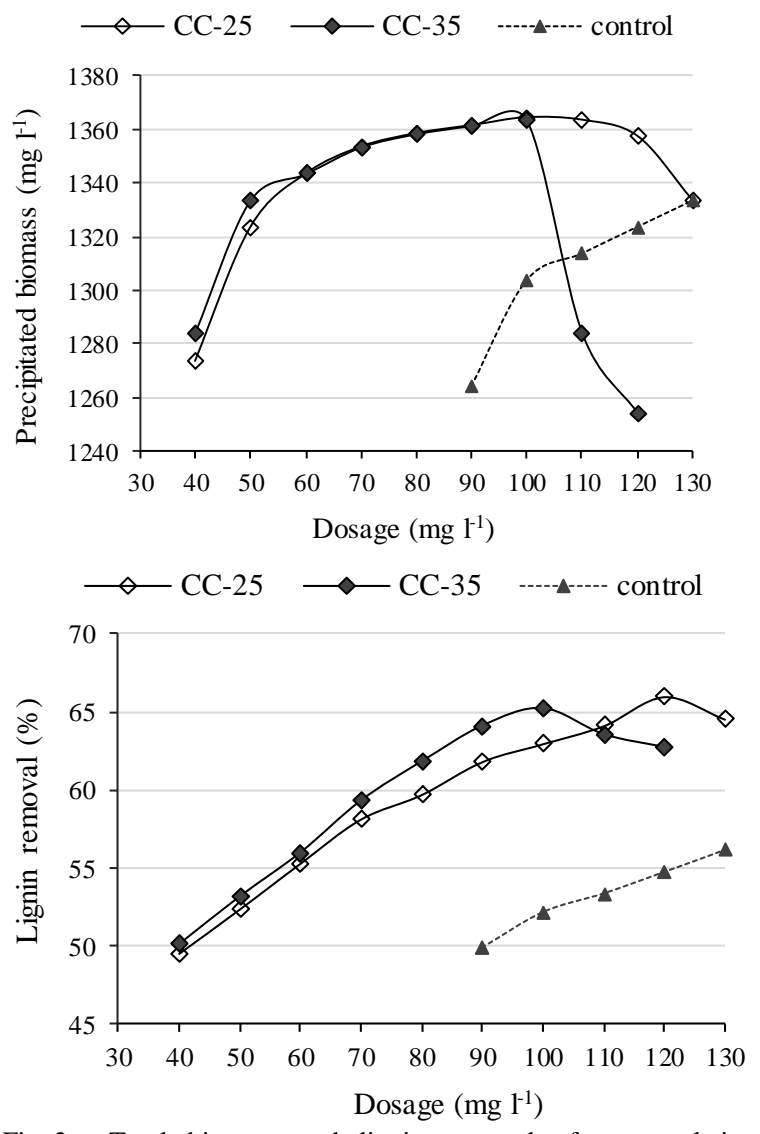

Fig. 2. Total biomass and lignin removal after coagulation treatment as a function of the dosage of $\mathrm{CC}(\mathrm{pH} 6)$.

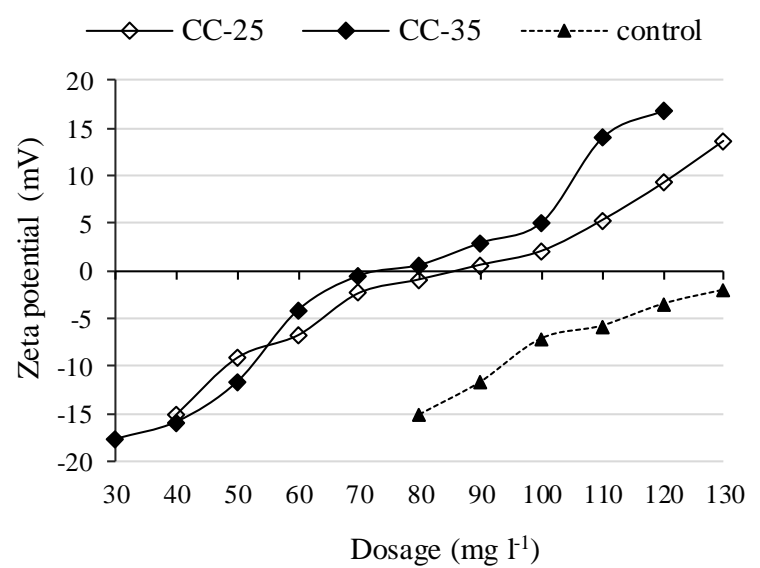

Fig. 3. Zeta potential of the model wastewater after coagulation as a function of coagulant dosage ( $\mathrm{pH} 6)$.

It is known that zeta potential values indicate the quality of the purification of wastewater. The variations of the zeta potential of the wastewater against the coagulant dosage after the coagulation treatment are shown in Fig. 3. It can be seen that the control coagulant gives a very slight decrease in the negative zeta potential values within the investigated dosages, while the zeta potential of CC-35 and CC-25 increases gradually and reaches the isoelectric points at a dosage of about 70 and $80 \mathrm{mgl}^{-1}$, respectively. 


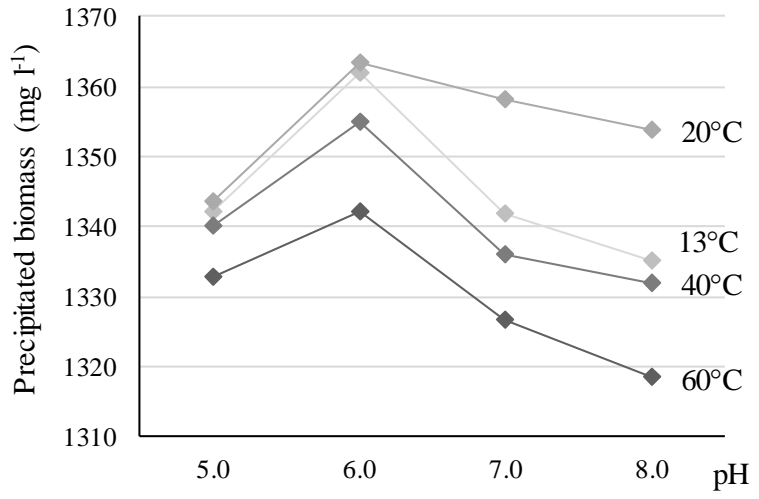

Fig. 4. Total biomass removal efficiency depending on the $\mathrm{pH}$ and temperature (CC-35, dosage $100 \mathrm{mgl}^{-1}$ ).

According to the obtained results, CC-35 was characterized by a higher total biomass and lignin removal from the wastewater as well as a lower optimal coagulation dosage.

The coagulation ability of CC-35 at different $\mathrm{pH}$ was also tested. The investigated $\mathrm{pH}$ range varied from 5 to 8 . It can be seen (Fig. 4) that the total biomass removal grows slightly from $\mathrm{pH} 5$ to 6 , but decreases towards the neutral and alkaline $\mathrm{pH}$ values. The optimal $\mathrm{pH}$ value for the biomass precipitation is pH 6.

Since the coagulation process is relatively sensitive to the coagulation temperature, it was of interest to compare the coagulation efficiency of CC35 in the temperature range of $13-60^{\circ} \mathrm{C}$. Fig. 4 shows that, over the whole temperature range, the $\mathrm{pH}$ relevance remains. The reduction of temperature below $20^{\circ} \mathrm{C}$ practically does not affect the effectiveness of the $\mathrm{CC}-35$ at the optimal $\mathrm{pH}$ 6. At the same time, with increasing temperature up to 40 and $60^{\circ} \mathrm{C}$, the biomass removal efficiency decreases.

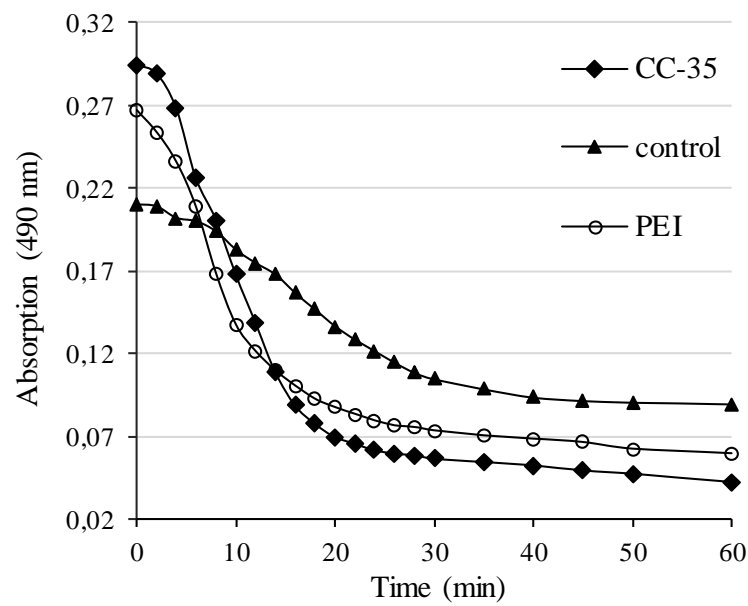

Fig. 5. Effect of coagulation time on the absorption $(490 \mathrm{~nm})$ of model wastewater in the coagulation process $(\mathrm{pH} 6$, dosage 100 $\left.\mathrm{mgl}^{-1}\right)$.

A study of sedimentation kinetics, using for this purpose the time-dependence of wastewater absorption at the wavelength $490 \mathrm{~nm}$, shows (Fig. 5) that the wastewater coagulated with $\mathrm{CC}-35$ has the highest turbidity in comparison with PEI and the control coagulant, and the coagulation process with the participation of CC-35 is completed practically during the first $30 \mathrm{~min}$. The diameter of the floccules obtained with CC-35 after $30 \mathrm{~min}$ of the coagulation was in a range of 1114-1242 nm, while these values for the floccules obtained with PEI and the control coagulant were significantly lower, in a range of 664842 and 331-499 nm, respectively.

Separated Lignocellulosic Biomass Characteristic

The previous studies [18] of the component composition of the biomass with instrumental analysis (FTIR-, UV-, Raman spectroscopy; HPLC) indicated that the dominant wood component in the obtained biomass were hemicelluloses, and the content of hemicelluloses, lignin and water-soluble degraded wood products in the biomass corresponded to the following mass ratio: $6.7 / 1.2 / 1.0$, respectively.

The study of the composition of the lignocelluloses by analytical pyrolysis indicates that the carbohydrates under investigation represent a mixture of low molecular wood degraded products, in which the content of cyclopentane derivatives is essential (Table 2).

Table 2

Products of carbohydrates pyrolysis

\begin{tabular}{ll}
\hline Cyclopentane derivatives & $55.60 \%$ \\
\hline $\begin{array}{l}\text { Aldehyde, Ketone } \\
\text { (Methylglyoxal and Acetone, }\end{array}$ & $25.35 \%$ \\
2-Butanone, 2-Pentanone, 2-Nonanone, & \\
2-Propanone, 1-hydroxy-2-Butanone, & \\
3-hydroxy-Hexanal) & $19.05 \%$ \\
\hline $\begin{array}{l}\text { Acid, Alcohol } \\
\text { (Acetic acid, 1,2-Ethanediol) }\end{array}$ \\
\hline
\end{tabular}

At lower temperatures, the thermal decomposition of carbohydrates starts with the depolymerization of the polymer and the cleavage of chemical bonds in the side chains. Therefore, the formation of acetic acid is involved with the primary elimination reaction of the active O-Ac groups in the side chain. At the higher pyrolysis temperature, the products are more complex, including aldehydes, ketones and alcohols from the oxygenation of $\mathrm{C} 2$ or $\mathrm{C} 3$ in the sugar ring.

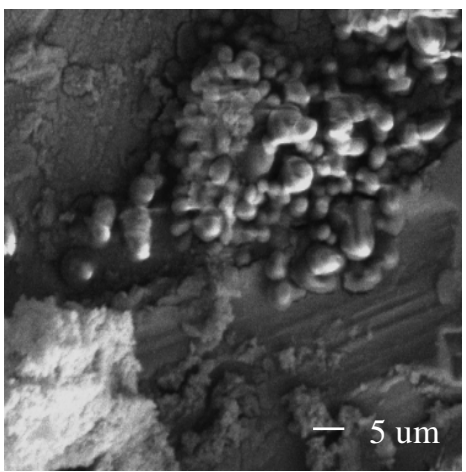

Fig. 6. SEM image of dried lignin and hemicelluloses coagulates. 
Environment. Technology. Resources, Rezekne, Latvia Proceedings of the $11^{\text {th }}$ International Scientific and Practical Conference. Volume III, 343-349

Precipitated Biomass as a Structuring Agent for Dust Suppression

The reduction in dust emission from unpaved roads can be achieved through the application of chemical dust suppressants. Chemical suppressants such as polymers act as adhesives to bond soil particles together. The resulting agglomerates of soil particles become too heavy to be uplifted and entrained in the atmosphere as dust.

The precipitated lignocellulosic biomass was studied as a structuring agent for dust suppression. The used biomass was applied in the gel form and was obtained by centrifugation of the precipitated biomass sludge without its drying. Stable suspensions with the defined biomass concentration were prepared by intensive mixing the biomass gel with water.

The fractional composition of the treated sandy aggregates is represented in Fig. 7. With growing content of the biomass $(0.2-0.8 \%)$, the amount of the sandy aggregates in the soil sample increases, and the values of the structure-forming coefficient K1 (Fig. 8) grow. Simultaneously, with increasing amount of the biomass, the structure-forming coefficient $\mathrm{K} 2$ also increases.

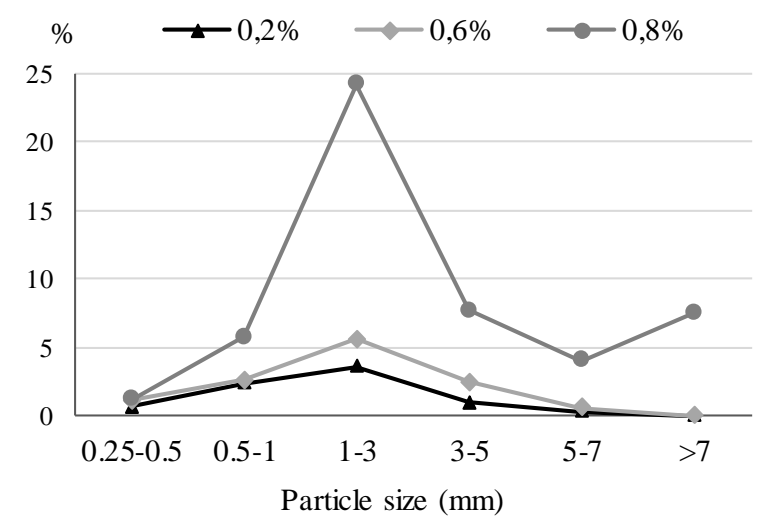

Fig. 7. Fractional composition of sandy soil depending on the added biomass content (100\% sand soil).

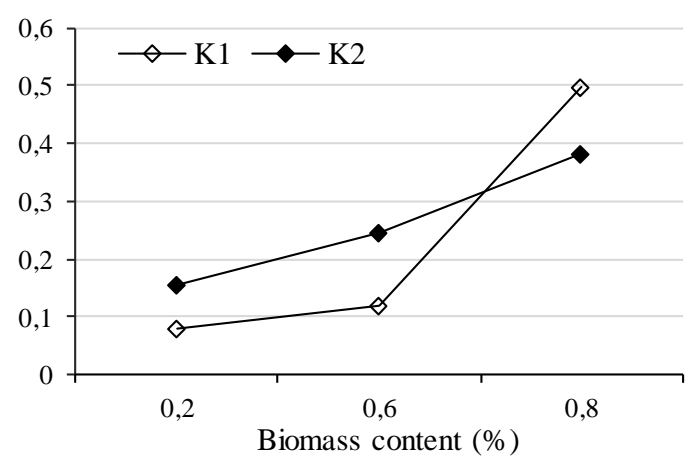

Fig. 8. Structuring coefficients $\mathrm{K} 1$ and $\mathrm{K} 2$ depending on the added biomass content (100\% sand soil).

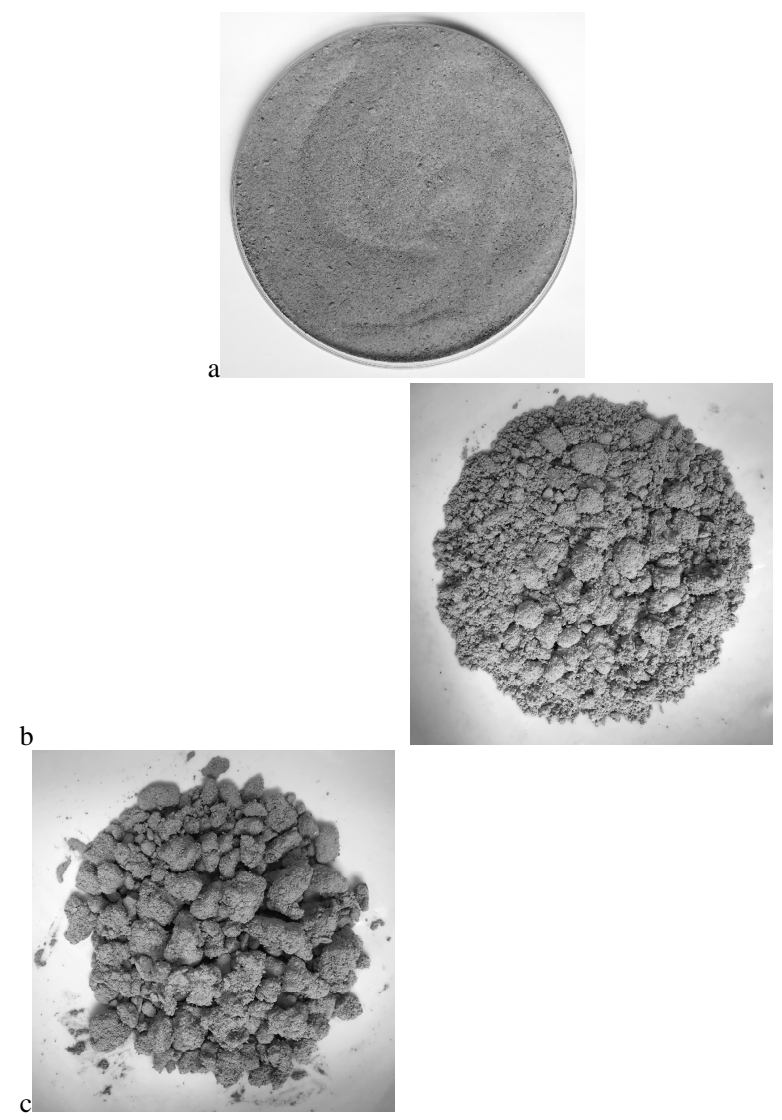

Fig. 9. Sand before (a) and after structuring with precipitated lignocellulosic biomass (b) $0.2 \%$; (c) $0.8 \%$.

Experiments were also conducted using powdered sand/clay model soil, varying the clay content within $0-70 \%$ (Fig. 10). Using $0.8 \%$ precipitated biomass as a structuring agent, the content and size of the aggregates increase with growing content of clay in the model soil. It verifies the increasing values of the structure-forming coefficients K1 and K2 (Fig. 11).

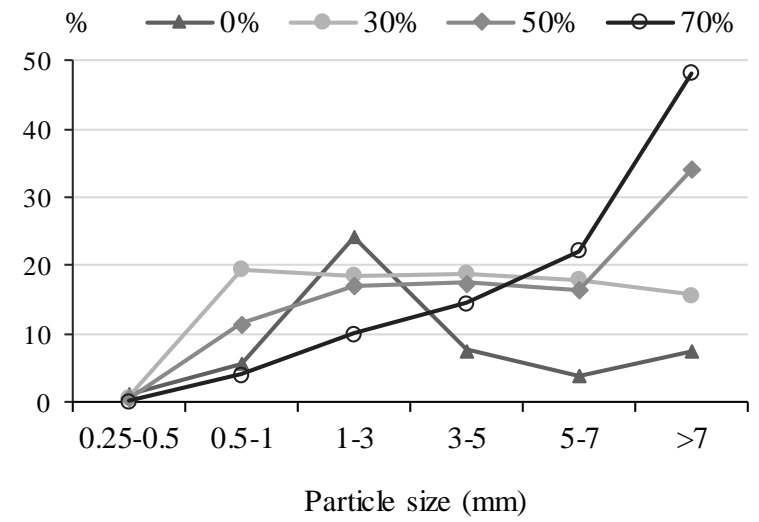

Fig. 10. Fractional composition of model soil depending on the clay content ( $0.8 \%$ biomass). 


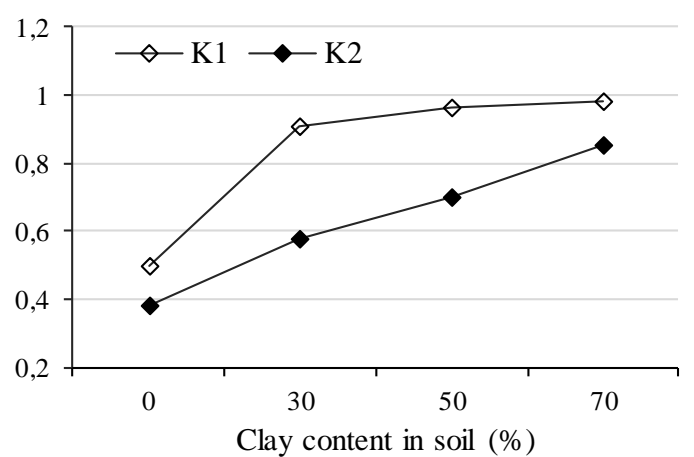

Fig. 11. Structuring coefficients K1 and K2 depending on the clay content in model soil ( $0.8 \%$ biomass).

The maximum amount of the aggregates (98 wt \%) is formed with the biomass content of $0.8 \%$ in model soil with the clay content of $70 \%$. The dust of the soil particles is exposed to an active transfer by the wind flow. The critical speed at which the movement of soil particles begins depends on the particle size.

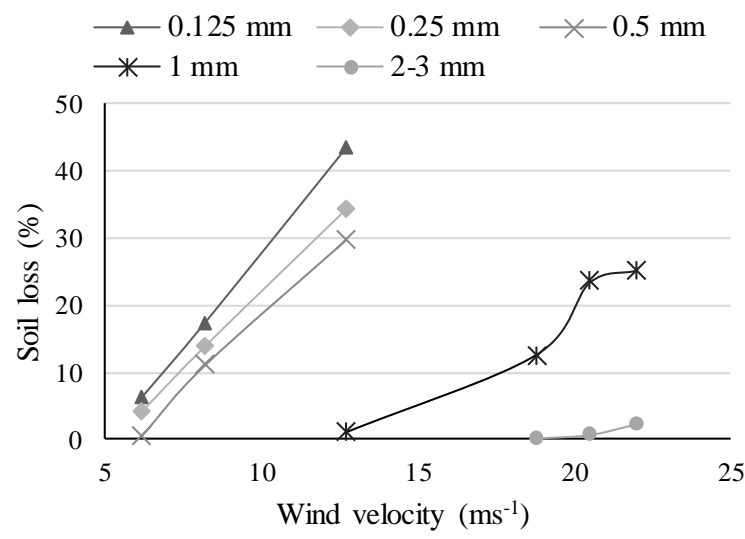

Fig. 12. Soil loss depending on the size of soil particles and wind velocity.

The obtained results show (Fig. 10) that the smaller the particle size, the greater the soil loss of the particles' mass. The movement of soil particles with the diameter below $0.50 \mathrm{~mm}$ starts at the wind velocity from $6 \mathrm{~ms}^{-1}$. The critical wind velocity for particles with a diameter of $1 \mathrm{~mm}$ is $12 \mathrm{~ms}^{-1}$. Increasing the velocity up to $22 \mathrm{~ms}^{-1}$, the soil loss does not exceed $25 \%$. As can be seen, most resistant against wind erosion are particles with a diameter above $2 \mathrm{~mm}$. The movement of the particles begins only when the wind velocity reaches $18 \mathrm{~ms}^{-1}$ and the loss is around $3 \%$.

\section{CONCLUSION}

Using the developed composite coagulant for recovery of birch wood biomass from plywood production model wastewater, at the optimal coagulation parameters, the separation of the total biomass achieves $97 \%$, but the extraction of lignin and lignin-like substances is more than $65 \%$.

The obtained results show that the separated lignocellulosic biomass is able to form large sandy aggregates, which are able to prevent the dusty soil blowing off from the unpaved road surface.

\section{V.ACKNOWLEDGMENTS}

The authors would like to thank the Ministry of Education and Science of the Republic of Latvia for the financial support of the National Research Programme (ResProd).

\section{REFERENCES}

[1] I. Mayer and G. Koch, "Element content and $\mathrm{pH}$ value in American black cherry (Prunus serotina) with regard to colour changes during heartwood formation and hot water treatment," Wood Sci. Technol., vol. 41, no. 6, 2007, pp. 537547.

[2] A. Rohumaa, A. Yamamoto, C. Hunt, C. Frihart, C. Hughes and J. Kers, "Effect of $\log$ soaking and the temperature of peeling on the properties of rotary-cut birch (Betula pendula Roth) veneer bonded with phenol-formaldehyde adhesive," Bioresources, vol. 11 no. 3, 2016, pp. 5829-5838.

[3] C. Hu, H. Liu, J. Qu, D. Wang and J. Ru, "Coagulation behaviour of aluminium salts in eutrophic water: significance of Al13 species and pH control," Environ. Sci. Technol., vol. 40, 2006, pp. 325-331

[4] T. Sun, L.-L. Liu, L.-L. Wan and Y.-P. Zhang, "Effect of silicon dose on preparation and coagulation performance of poly-ferric-aluminum-silicate-sulfate from oil shale ash," Chem. Eng. J. vol. 163, 2010, pp. 48-54.

[5] S. Chakrabarti, S. Banerjee, B. Chaudhuri, S. Bhattacharjee and B.K. Dutta, "Application of biodegradable natural polymers for flocculated sedimentation of clay slurry," Bioresour. Technol., vol. 99, 2008, pp. 3313-3317.

[6] W. Brostow, H. E. H. Lobland, S. Pal and R. P. Singh, "Polymeric flocculants for wastewater and industrial effluent treatment," J. Mater. Ed., vol. 31, 2009, pp. 157-166.

[7] K. E. Lee, N. Morad, T. T. Teng and B. T. Poh, "Development, characterization and the application of hybrid materials in coagulation/flocculation of wastewater: A review," Chem. Eng. J., vol. 203, 2012, pp. 370-386.

[8] T. Ahmad, K. Ahmad and M. Alam, "Sustainable management of water treatment sludge through $3^{\text {' }} \mathrm{R}$ ' concept," Journal of Cleaner Production, vol. 124, 2016, pp. $1-13$.

[9] C. Huang, J. R. Pan, K. D. Sun and C. T. Liaw, "Reuse of water treatment plant sludge and dam sediment in brick making," Water Sci. Technol., vol. 44, no. 10, 2001, pp. 273277.

[10] O. Kizinievic, R. Zurauskiene, V. Kizinievic and R. Zurauskas, "Utilisation of sludge waste from water treatment for ceramic products," Constr. Build. Mater. vol. 41, 2013, pp. 464-473.

[11] Q. C. Nowasell and J. T. Kevern, "Using drinking water treatment waste as a lowcost internal curing agent for concrete," ACI Mater. J., vol. 112, no. 1, 2015, pp. 69-78.

[12] J. R. Pan, C. Huang and S. Lin, "Reuse of fresh water sludge in cement making," Water Sci. Technol., vol. 50, no. 9, 2004, pp. 183-188.

[13] C.-H. Huang and S.-Y. Wang, "Application of water treatment sludge in the manufacturing of lightweight aggregate," Constr. Build. Mater., vol. 43, 2013, pp. 174-183.

[14] L. Yang, J. Wei, Y. Zhang, J. Wang and D. Wang, "Reuse of acid coagulant recovered drinking waterworks sludge residual to remove phosphorus from wastewater," Appl. Surf. Sci., vol. 305, 2014, pp. 337-346.

[15] K. C. Makris, D. Sarkar and R. Datta, "Evaluating a drinking water waste byproduct as a novel sorbent for arsenic," Chemosphere, vol. 64, 2006, pp. 730-741.

[16] L. W. Titshall and J. C. Hughes, "Characterization of some South African water treatment residues and implications for land application," J. Water SA, vol. 31, no. 3, 2005, pp. 299307. 
Environment. Technology. Resources, Rezekne, Latvia Proceedings of the $11^{\text {th }}$ International Scientific and Practical Conference. Volume III, 343-349

[17] E. A. Dayton and N. T. Basta, "Characterization of drinking water treatment residuals for use as a soil substitute," Water. Environ. Res., vol. 73, no. 1, 2001, pp. 52-57.

[18] G. Shulga, S. Vitolina, J. Brovkina, B. Neiberte, A Verovkins, M. Puḳe and N. Vedernikovs, "Wood biomass from the model wastewater and its fractionation," in Proceedings of the $9^{\text {th }}$ International Scientific and Practical Conference. Environment. Technology. Resources, Vol. 1, 2013, pp. 190-194.
[19] G. Shulga, J. Brovkina, B. Neiberte, J. Ozoliņš and R. Neilands, "A method for wastewater treating from lignin and hemicellulose substances at wood processing plants," LV Patent 14789A, January 20, 2014

[20] Z. Liu, Y. Ni, P. Fatehi and A. Saeed, "Isolation and cationization of hemicelluloses from pre-hydrolysis liquor of kraft-based dissolving pulp production process," Biomass Bioenerg., vol. 35, 2011, pp. 1789-1796. 